\title{
Gain Reduction Measurements in Transient Stimulated Raman Scattering
}

\author{
R. J. Heeman and H. P. Godfried
}

\begin{abstract}
Threshold energy measurements of transient rotational Stimulated Raman Scattering are compared to Raman conversion calculations from semiclassical theories using a simple concept of a gain reduction factor which expresses the reduction of the gain from its steady-state value due to transient scattering. Excellent agreement was found with theoretically derived gain reduction in the transient and highly transient case.
\end{abstract}

\section{INTRODUCTION}

S TIMULATED Raman Scattering (SRS) can be an efficient way to generate new optical frequencies. Frequently, however, high pump intensities are required for efficient scattering of the incoming radiation. Such high intensities may be obtained by focusing the radiation from high power pulsed lasers. If the pump pulse width is comparable to the dephasing time of the Raman polarizability, the scattering is transient, which results in reduced conversion efficiencies and increased Raman thresholds, due to a decrease in the Raman gain with respect to its steady-state value. In the present work, the transient character of the scattering is described by a gain reduction factor by which the steadystate Raman gain is reduced. Previously [1], the gain reduction was used to compare threshold measurements to transient theory. Agreement was found for experiments performed with parallel beams, while measurements from focused experiments did not agree. In the experiments described in this paper, focused beams were used. A short Nd-YAG pump pulse was scattered transiently in a multipass cell at room temperature filled with molecular hydrogen gas. The gain reduction was derived from measurements of the threshold energy as a function of the pump pulse width and pressure. The advantage of threshold experiments is the constant power gain determined by the Stokes noise power and the chosen Stokes power threshold level. Additionally, pump depletion does not occur at threshold, which makes it easier to compare the measured gain reduction to the gain reduction calculated from theory.

In Section II, transient SRS is discussed briefly and the gain reduction is introduced phenomenologically. In Section III, the experimental setup is described. The analysis of the threshold experiments and the results are presented in Section IV. These results are discussed and compared to theory in Section V.

Manuscript received May 9, 1994; revised August 19, 1994. The work of R. J. Heeman was supported in part by the FOM (Foundation for Fundamental Research on Matter).

R. J. Heeman is with the Department of Applied Physics, University of Twente, $7500 \mathrm{AE}$ Enschede. The Netherlands.

H. P. Godfried is with the Nederlands Centrum voor Laser Research (NCLR), 7500 CR Enschede. The Netherlands.

IEEE Log Number 9407780.

\section{THEORY}

\section{A. Transient SRS}

For plane pump and Stokes waves with equal group velocities, SRS is described as a coupling of the optical waves to the Raman polarizability or coherent material excitation [2]. In a moving frame $\left(z^{\prime}, t^{\prime}\right)$ where the optical pulses are at rest, the set of coupled equation becomes [3]

$$
\begin{gathered}
\frac{\partial}{\partial z^{\prime}} E_{p}\left(z^{\prime}, t^{\prime}\right)=-K_{p} E_{s} A_{Q} \\
\frac{\partial}{\partial z^{\prime}} E_{s}\left(z^{\prime}, t^{\prime}\right)=K_{s} E_{p} A_{Q}^{*} \\
\left(\frac{\partial}{\partial t^{\prime}}+\Gamma\right) A_{Q}^{*}=E_{p}^{*} E_{s} .
\end{gathered}
$$

$K_{p}$ and $K_{s}$ are coupling constants which include the Raman susceptibility; $E_{p}$ and $E_{s}$ are the pump and Stokes optical field amplitudes, respectively; and $A_{Q}$ is the amplitude of the material excitation. The dephasing time is given by $(\pi \Gamma)^{-1}$ where $\Gamma$ is the Raman linewidth.

In transient SRS, the pulse width becomes comparable to the dephasing time of the material excitation. With the assumption that the pump pulse is not depleted, $\partial E_{p} / \partial z^{\prime}=0$, only two equations are left to be solved. They are first reduced to a second-order partial differential equation by writing $U=$ $F e^{\Gamma t^{\prime}}[3]$, where $F$ may be either $A_{Q}$ or $E_{s}$

$$
\left(\frac{\partial^{2}}{\partial t^{\prime} \partial z^{\prime}}-K_{s}\left|E_{p}\left(t^{\prime}\right)\right|^{2}\right) U=0 .
$$

If, furthermore, the variable $\tau$ is introduced, $\tau=$ $\int_{-\infty}^{t^{\prime}}\left|E_{p}\left(t^{\prime \prime}\right)\right|^{2} d t^{\prime \prime}$, the partial differential equation can be written in the standard form of a hyperbolic equation

$$
\left(\frac{\partial^{2}}{\partial \tau \partial z^{\prime}}-K_{s}\right) U=0
$$

The integral $\tau$ gives the total energy of the pump pulse up to a time $t^{\prime}$. The solution for the Stokes field in forward Raman scattering takes the form [2]-[6]

$$
\begin{aligned}
E_{s}\left(z, t^{\prime}\right)= & E_{s}\left(0, t^{\prime}\right)+\left(K_{s} z\right)^{1 / 2} E_{p}\left(t^{\prime}\right) \\
& \cdot \int_{-\infty}^{t^{\prime}} e^{-\Gamma\left(t^{\prime}-t^{\prime \prime}\right)} \frac{E_{p}^{*}\left(t^{\prime \prime}\right) E_{s}\left(0, t^{\prime \prime}\right)}{\left(\tau\left(t^{\prime}\right)-\tau\left(t^{\prime \prime}\right)\right)^{1 / 2}} \\
& \cdot I_{1}\left[2\left(K_{s} z\left(\tau\left(t^{\prime}\right)-\tau\left(t^{\prime \prime}\right)\right)\right)^{1 / 2}\right] d t^{\prime \prime}
\end{aligned}
$$

where $z$ is written instead of $z^{\prime}$ for convenience. It is assumed that at $t^{\prime} \rightarrow-\infty$, no material excitation exists, and that at 
$z=0$ a finite input Stokes field $E_{s}\left(0, t^{\prime}\right)$ may be present, $I_{1}$ is the first-order modified Bessel function [7]. After a linear growth for small $z\left(I_{1}(x) \simeq x\right.$ for $x \ll 1$, where $x$ is the argument of the Bessel function), the Stokes amplitude grows exponentially. In the limit of high gain $g_{s} z \cdot \Gamma t^{\prime} \gg 1$, solutions can be found for both the steady-state and transient limits. In the steady-state limit $\Gamma t^{\prime} \gg 1$, the solution for the Stokes amplitude is exponential with a plane wave gain coefficient given by $g_{s}=K_{s} \Gamma^{-1}\left|E_{p}\right|^{2}$. This solution is also readily obtained from the coupled set (1) when it is noted that, in steady state, no changes in $A_{Q}$ occur for a constant pump: $\partial A_{Q} / \partial t=0$. The criterion $\Gamma t^{\prime} \gg 1$ is rather strong and can be relaxed to [3], [5]

$$
\Gamma t^{\prime}>2 g_{s} z
$$

for which the steady-state solution is still well approximated. In the highly transient regime, $\Gamma t^{\prime} \ll 1$, the exponent $-\Gamma\left(t^{\prime}-\right.$ $\left.t^{\prime \prime}\right)$ in (4) can be neglected. The Stokes gain is then determined by the asymptotic behavior of the modified Bessel function $I_{1}(x) \simeq(2 \pi x)^{-1 / 2} e^{x}$ for $x \gg 1$. This results in the transient amplitude gain [3]

$$
g_{t} z=\ln \left[\frac{\left|E_{s}\right|}{\left|E_{s 0}\right|}\right] \cong 2\left(K_{s} z \int_{-\infty}^{t^{\prime}}\left|E_{p}\left(t^{\prime \prime}\right)\right|^{2} d t^{\prime \prime}\right)^{1 / 2}
$$

which is often written as [3], [5], [6]

$$
g_{t} z \simeq 2\left(K_{s} z\left|E_{p}\right|^{2} t^{\prime}\right)^{1 / 2}=2\left(g_{s} z \Gamma t^{\prime}\right)^{1 / 2}
$$

where a rectangular pump pulse of width $t^{\prime}$ is used to calculate the integral. The condition $\Gamma t^{\prime} \ll 1$ may be somewhat relaxed to $\Gamma t^{\prime}<g_{s} z$. Then, Wang [4] found for the Stokes amplitude in this transient regime

$$
E_{s} \simeq E_{s 0} \cdot \frac{e^{a \tau_{0}-\tau_{0}^{2}}}{\left(4 \pi a \tau_{0}\right)^{1 / 2}}
$$

where $a$ and $\tau_{0}$ are given by $a^{2}=4 g_{s} z$ and $\tau_{0}^{2}=\Gamma t^{\prime}$, respectively. As mentioned above, these solutions were obtained assuming plane waves and no pump depletion.

\section{B. Focusing Properties in SRS}

Since a multipass cell was used in which a pump beam is focused in each pass, the result of scattering in a focused zeroth-order Gaussian beam is discussed briefly. An approximate solution for steady-state Raman scattering has been given previously [8], [9]. The Stokes power gain per transit through one focus $P_{s}=P_{s 0} \cdot e^{\alpha_{1}}$ is given by

$$
\alpha_{1}=\frac{4 P_{p} G_{s}}{\lambda_{p}+\lambda_{s}} \cdot \tan ^{-1}\left(\frac{L}{b}\right)
$$

where $G_{s}$ is the steady-state plane wave intensity gain coefficient; $\lambda_{p}$ and $\lambda_{s}$ the pump and Stokes wavelengths, respectively; and $P_{p}$ is the pump power. $L$ is the cell length, and $b$ is the confocal parameter $b=2 \pi \omega_{0}^{2} / \lambda$, with $\omega_{0}$ the beam waist and $\lambda$ the wavelength. It is seen that tight focusing increases the Raman gain, approaching maximum when $\tan ^{-1}(L / b)$ approaches $\pi / 2$. Beyond this, only higher pump powers increase the Raman gain. The power gain depends on the wavelength as $\left(\lambda_{s}\left(\lambda_{p}+\lambda_{s}\right)\right)^{-1}$, since $G_{s}$ is proportional to $\lambda_{s}^{-1}$ [10]. For long Stokes wavelengths, this results in a low power gain. When the pump laser beam is repeatedly focused in a multipass cell, the power gain is further enhanced. The power gain $\alpha_{N}$ after $N$ passes becomes [11]

$$
\alpha_{N}=\frac{1-r^{N}}{1-r} \cdot \alpha_{1}+(N-1) \cdot \ln (r)
$$

where $r$ is the intensity reflectivity of the multipass cell mirrors, and $\alpha_{1}$ the single focus power gain coefficient. In this approach, transient scattering could be accounted for phenomenologically when the steady-state gain coefficient $G_{s}$ is replaced by the reduced gain $R \cdot G_{s}$, where $R$ is the gain reduction. The gain reduction in this form is introduced without firm arguments. As is shown later on, its justification is found experimentally.

\section{EXPERIMENTAL SETUP}

In the experiments, a $Q$-switched Nd-YAG pump laser was used. This laser was injection seeded and produced a single longitudinal mode pulse. The pulse width could be varied between 10 and approximately 60 ns FWHM by an externally applied $Q$-switch delay, and was measured from the pulse shapes recorded with an $\mathrm{Si}$ photodiode. Its pulse energy was measured with a calibrated pyroelectric detector. The NdYAG beam was first Fourier filtered to improve the transverse beam profile, and then mode matched to the transverse mode of the multipass Raman cell. The pump power level was adjusted by rotating the linear polarization of the pump laser radiation relative to a set of two dielectric polarizers. Neither the $Q$-switch nor the lamp pump power settings were used, because both change the beam mode and the pulse width which influence the scattering behavior. Adjusting the pump power level by rotating the polarization was done with special care. Due to thermal gradients in the optically pumped Nd-YAG rods of the oscillator and amplifier, stress-induced birefringence [12] partially depolarizes the beam. This changes the beam's transverse intensity profile when passing through the polarizers. As a result, the pump beam may contain higher order modes which may experience losses due to diffraction on the mirrors and windows of the multipass Raman cell. This effect would show up as a rise of the Raman threshold power with respect to an unaffected threshold curve. To obtain a Gaussian beam cross section and a low adjustable power level, partially reflecting mirrors reduced the power to a level in the range where the polarizers could be used. The linear polarization of the Nd-YAG laser beam was changed to circular to maximize the Raman gain [13]. A half- and a quarter-wave plate were used rather than a single quarterwave plate to be able to correct for the small polarization changes due to reflections on folding copper mirrors. These were used at large angles of incidence to inject the $\mathrm{Nd}$ YAG laser beam into the multipass Raman cell. The beam polarization was fixed in all experiments and carefully adjusted to be circular, using the known polarization properties of SRS for low pump intensities. In contrast to a linear polarized pump, a circularly polarized pump does not generate antiStokes radiation through Stokes anti-Stokes coupling [1], [13], 
[14] unless, at higher intensities, second-order Stokes light is generated by Raman scattering from first Stokes radiation and back conversion by four wave mixing processes produces anti-Stokes radiation. In the experiments, a multipass Raman cell was used. This has the advantage over a single pass cell of allowing a moderate gain per pass. The scattered radiation transverse intensity distribution is then very nearly a Gaussian as assumed in (9). This allows a comparison to theory. In the high gain case, on the other hand, gain guiding [15] would become important and (9) would no longer be valid. The Herriott-type [16] multipass cell is of conventional design [8]. It consists of two spherical mirrors in a near concentric stable configuration in which a mode matched beam reproduces its transverse shape on each pass through the cell. This is also the case when the beam is injected off axis. By properly adjusting the angle at which the beam is injected, the reflection spots on each mirror can be made to lie on a circle. The cell was filled with molecular hydrogen gas, which was either the paramodification or the normal (statistical) mixture of ortho- and parahydrogen, at pressures between 0.1 and 2 bar. In this pressure range, the dephasing time of the Raman polarizability is a few nanoseconds (approximately $3 \mathrm{~ns}$ at 1 bar). The threshold condition was defined by the smallest Stokes peak power (typically $1.4 \mathrm{~kW}$ ), detectable in the setup where a fast InGaAs PIN photodiode was used. The pump power which generates this Stokes power level was taken as the threshold power. Although the Stokes power level at threshold condition is arbitrarily chosen, the threshold power itself is well defined due to the fast growing Stokes power for nondepleted pump waves. The power gain at threshold is thus fixed at $2 g z=\ln \left(P_{s, t h} / P_{s, 0}\right) \simeq 32$, where the noise Stokes power $P_{s, 0}$ is given by $h \nu_{s} \Gamma \simeq 10^{-11} \mathrm{~W}$.

\section{ANALYSIS AND RESULTS OF THE THRESHOLD EXPERIMENTS}

\section{A. Gain Reduction}

Scattering becomes transient when the interaction time of the pump wave with the local medium is of the same order of magnitude as the medium dephasing time. The dephasing time is dependent on pressure and is well determined by $(\pi \Gamma)^{-1}$ at high gas pressures. At lower pressures, however, the Raman linewidth is influenced by Doppler broadening, and possibly Dicke narrowing [17], [18] will occur. Then the line shapes change from Lorentzian to Doppler, and consequently the dephasing time cannot be defined. Therefore, the product of the Raman linewidth and the interaction time $\Gamma(p) \cdot t^{\prime}$ is used to define the transient regime rather than the ratio of the interaction time and the dephasing time. The reduction of the steady-state Raman gain due to transient scattering may thus be defined as a function of this product. Due to this definition, the same gain reduction may occur for two experimental conditions $\left(p \cdot t^{\prime}\right)$ if the product $\Gamma(p) \cdot t^{\prime}$ is equal, i.e., $R_{1}=R_{2}$ for

$$
\Gamma\left(p_{1}\right) \cdot t_{1}^{\prime}=\Gamma\left(p_{2}\right) \cdot t_{2}^{\prime} .
$$

In the threshold experiments described in this paper, where the total pump energy is measured, the interaction time $t^{\prime}$ is defined as the pump pulse width at half height. Therefore, $t^{\prime}$ is written for the pulse width measured in the experiments. The linewidth $\Gamma(p)$ is pressure dependent and is well described by $\Gamma(p)=B \cdot p$ at high pressures where the Raman linewidth is pressure broadened.

The scattering experiments performed at constant pressure $p_{0}$ become transient for short interaction times. To describe transient scattering in focused Gaussian beams, the gain reduction factor introduced in Section II is used. Then the single pass power gain (9) at threshold condition becomes

$$
\alpha_{1}=\frac{4 P_{t h} R G_{s}}{\lambda_{p}+\lambda_{s}} \cdot \tan ^{-1}\left(\frac{L}{b}\right)
$$

where $P_{t h}$ is the pump power to obtain the threshold condition. The product $P_{t h} R G_{s}$ is a constant at threshold and must equal $P_{t h, s} G_{s}$ for the same threshold condition at steady state. $R$ is thus found from the threshold experiments performed at constant pressure $p_{0}$ as

$$
R\left(p_{0} . t_{0}^{\prime}\right)=\frac{P_{t h, s}\left(p_{0}\right)}{P_{t h}\left(p_{0} \cdot t_{0}^{\prime}\right)}=\frac{P_{t h}\left(p_{0}, t_{0}^{\prime} \rightarrow \infty\right)}{P_{t h}\left(p_{0}, t_{0}^{\prime}\right)}
$$

where $P_{t h . s}\left(p_{0}\right)$ is the steady-state threshold power at pressure $p_{0}$. The threshold experiments are performed as a function of pressure $p$ at a fixed pulse width $t^{\prime}$, and as a function of pulse width $t_{0}^{\prime}$ at a fixed pressure $p_{0}$. These two experimental conditions are connected by (11).

The measured threshold energy is shown in Fig. 1(a) as a function of pulse width $t_{0}^{\prime}$, and in Fig. 1(b) as a function of pressure $p$. Although the energy data in Fig. 1(a) show some curvature, an empirical, linear fitting function was used, the slope of which equals the steady-state threshold power

$$
E_{t h}\left(p_{0}, t_{0}^{\prime}\right)=E_{o}\left(p_{0}\right)+P_{t h, s}\left(p_{0}\right) \cdot t_{0}^{\prime} .
$$

The gain reduction equation (13) is thus written as

$$
R\left(p_{0}, t_{0}^{\prime}\right)=\left(1+\frac{E_{o}\left(p_{0}\right)}{P_{t h, s}\left(p_{0}\right) t_{0}^{\prime}}\right)^{-1}
$$

where $P_{t h}\left(p_{0}, t_{0}^{\prime}\right)=E_{t h}\left(p_{0}, t_{0}^{\prime}\right) / t_{0}^{\prime}$ is used. With (11) to express $t_{0}^{\prime}$ in terms of $t^{\prime}, \Gamma(p)$ and $\Gamma\left(p_{0}\right)$, the gain reduction becomes

$$
R\left(\Gamma(p) \cdot t^{\prime}\right)=\left(1+\frac{E_{o}\left(p_{0}\right) \Gamma\left(p_{0}\right)}{P_{t h, s}\left(p_{0}\right) \Gamma(p) \cdot t^{\prime}}\right)^{-1} .
$$

This concept, in which a function of $\Gamma \cdot t^{\prime}$ is derived from threshold measurements, readily allows comparison to transient SRS theories. The fitting parameters $E_{o}\left(p_{0}\right)$ and $P_{t h, s}\left(p_{0}\right)$ at a pressure $p_{0}=1.2$ bar are given in Fig. 1(a). In the threshold experiments, where the pressure was varied, the pulse width $t^{\prime}$ was $21 \mathrm{~ns}$. Still, $\Gamma\left(p_{0}\right)$ must be determined. For pressures above 2 bar, the Raman linewidth is well determined by pressure broadening, $\Gamma(p)=B \cdot p$, where $B$ is the pressure broadening coefficient. For lower pressures, the Raman linewidth is not exactly known since Doppler broadening, pressure broadening, and possibly Dicke narrowing influences the linewidth. As is shown below, the same threshold measurements can be used to determine the Raman linewidth. 


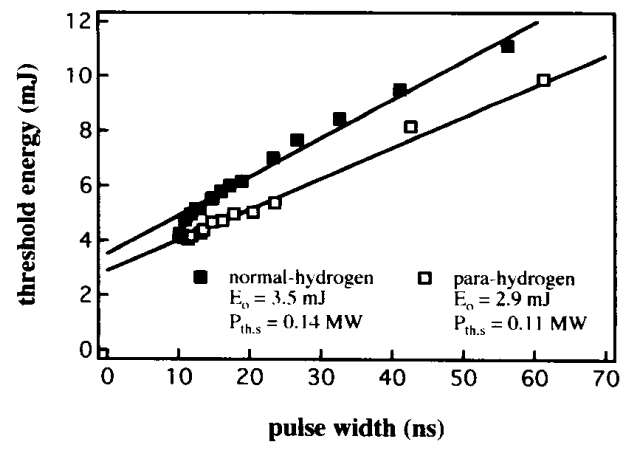

(a)

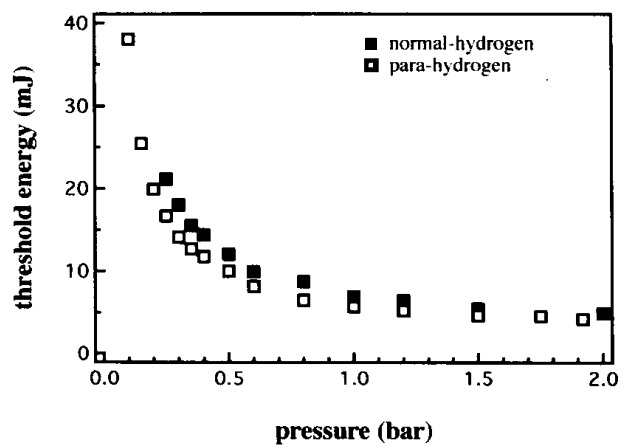

(b)

Fig. 1. Measured threshold energy in $n-H_{2}(\square)$ and $p-H_{2}(\square)$ as a function of pulse width at 1.2 bar gas pressure (a), and as a function of pressure for a pulse width of $21 \mathrm{~ns}(\mathrm{~b})$.

\section{B. Determination of the Raman Linewidth}

The Raman linewidth was determined for pressures for which the threshold measurements were made, i.e., between 0.1 and 2 bar. The pressure dependence of the steady-state Raman gain can be expressed as [10]

$$
\frac{G_{s}\left(p_{1}\right)}{G_{s}\left(p_{2}\right)}=\frac{p_{1} / \Gamma\left(p_{1}\right)}{p_{2} / \Gamma\left(p_{2}\right)} .
$$

At threshold, the power gain is a constant. Writing the relevant parameters only, the constant power gain for the two experimental conditions at threshold implies

$$
\begin{aligned}
P_{t h}\left(p_{0}, t_{0}^{\prime}\right) \cdot R\left(\Gamma\left(p_{0}\right) \cdot t_{0}^{\prime}\right) \cdot G_{s}\left(p_{0}\right) \\
\quad=P_{t h}\left(p, t^{\prime}\right) \cdot R\left(\Gamma(p) \cdot t^{\prime}\right) \cdot G_{s}(p) .
\end{aligned}
$$

Combining (17) and (18), the Raman linewidth at pressure $p$ is expressed in terms of the Raman linewidth at pressure $p_{0}$ as

$$
\Gamma(p)=\frac{R\left(\Gamma(p) \cdot t^{\prime}\right) \cdot P_{t h}\left(p, t^{\prime}\right)}{P_{t h, s}\left(p_{0}\right)} \cdot \frac{p}{p_{0}} \cdot \Gamma\left(p_{0}\right)
$$

where $P_{t h, s}\left(p_{0}\right)=R\left(\Gamma\left(p_{0}\right) \cdot t_{0}^{\prime}\right) \cdot P_{t h}\left(p_{0}, t_{0}^{\prime}\right)$ is used, and which is found as the slope of the linear fit on the threshold measurements versus pulse width performed at a fixed pressure $p_{0}$. The threshold power $P_{t h}\left(p, t^{\prime}\right)$ was measured versus pressure for a fixed pulse width $t^{\prime}$. The calculation of the Raman linewidth becomes an iteration because the gain reduction still depends on the Raman linewidth. As a starting value, the pressure broadened linewidth $\Gamma(p)=B \cdot p$ is first used to determine the gain reduction with (16). Then the linewidth is calculated with (19) and used to determine the gain reduction again, etc., until the iteration converges. It is to be noted that (19) still contains $\Gamma\left(p_{0}\right)$, the Raman linewidth at pressure $p_{0}$. So, strictly speaking, only a relative measurement can be made in this way. As an extra constraint, the known high-pressure behavior of the linewidth $\Gamma(p)=B \cdot p$ may be used. The chosen value of $\Gamma\left(p_{0}\right)$ which gives the correct linewidth behavior at high pressures, determines the low pressure behavior.

At low pressures, on each successive iteration, the Raman linewidth departs more from the pressure broadened linewidth. Within approximately 40 steps, the linewidth converges to less than $1 \%$. Fig. 2 shows the derived Raman linewidth which is matched to the known high pressure behavior. It appears that $\Gamma\left(p_{0}\right)$ can be readily determined. Changing $\Gamma\left(p_{0}\right)$ results in changes of the high-pressure slope. Therefore, the values of the linewidth at low pressures vary only slightly. Adjusting $\Gamma\left(p_{0}\right)$ to obtain the correct high-pressure slope, $\Gamma\left(p_{0}\right)$ is found with small error. At a gas pressure of 1.2 bar, the Raman linewidth becomes $(118 \pm 5) \mathrm{MHz}$ for the $S_{0}(0)$ transition in $\mathrm{p}-\mathrm{H}_{2}$ and $(121 \pm 5) \mathrm{MHz}$ for the $S_{0}(1)$ transition in $\mathrm{n}-\mathrm{H}_{2}$. At lower pressures, the smallest derived Raman linewidth is $40 \mathrm{MHz}$ for the $S_{0}(1)$ transition in $\mathrm{n}-\mathrm{H}_{2}$ and $20 \mathrm{MHz}$ for the $S_{0}(0)$ transition in $\mathrm{p}-\mathrm{H}_{2}$, which is far below the Doppler linewidths of 153 and $91.8 \mathrm{MHz}$, respectively [19]. So, at room temperature, both $\mathrm{n}-\mathrm{H}_{2}$ and $\mathrm{p}-\mathrm{H}_{2}$ have a Dicke minimum in the $S_{0}(1)$ and the $S_{0}(0)$ transition, respectively, which is slightly deeper than predicted by the sum of a diffusion limited (or Dicke narrowed) and a pressure broadened linewidth [20]

$$
\Gamma(p)=\frac{k_{R}^{2} D_{0}}{\pi p}+B \cdot p .
$$

Here, $D_{0}$ is the seif-diffusion constant $D_{0}=1.49 \mathrm{~cm}^{2} \cdot \mathrm{bar} / \mathrm{s}$ [20], $B$ is the pressure broadening coefficient $B=97.3$ $\mathrm{MHz} / \mathrm{bar}$ [21], and $k_{R}$ is the difference between the pump and the Stokes wave vectors. In Fig. 2, the dotted curve is calculated by (20). Somewhat better agreement is obtained with the empiric formula

$$
\Gamma(p)=\frac{k_{R}^{2} D_{0}}{\pi(p+\rho)}+B \cdot p
$$

where parameter $\rho$ is chosen such that the correct zero pressure Doppler limit is obtained. This is indicated by the dashed curve in Fig. 2. For the $S_{0}(0)$ transition in $\mathrm{p}-\mathrm{H}_{2}$ and the $S_{0}(1)$ transition in $\mathrm{n}-\mathrm{H}_{2}, \rho$ is found to be $2.6 \cdot 10^{-2}$ bar and $4.2 \cdot 10^{-2}$ bar, respectively. The ratio of these values equals the ratio of the Raman shifts in $\mathrm{n}-\mathrm{H}_{2}$ and $\mathrm{p}-\mathrm{H}_{2}$, since the Doppler linewidths are proportional to these quantities. It is to be noted that (21) gives a finite zero pressure slope instead of zero, as it should be.

\section{Discussion}

The gain reduction as a function of $\Gamma t^{\prime}$ is readily obtained by (16) in the range $1<\Gamma t^{\prime}<8$, where the threshold experiments are performed. It is plotted in Fig. 3 as the curves (a) and (b) 


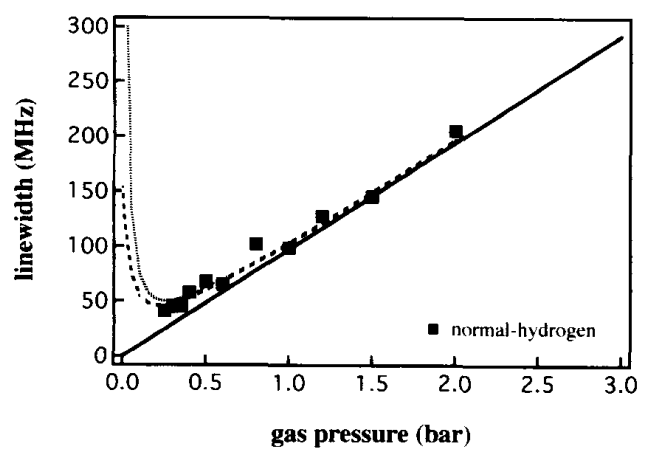

(a)

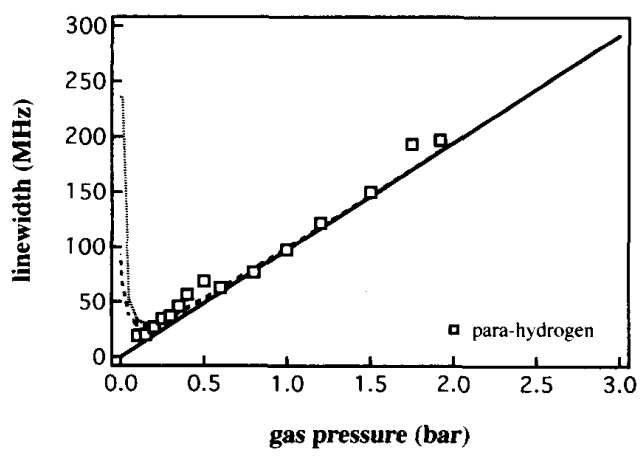

(b)

Fig. 2. Raman linewidth as a function of gas pressure derived from the threshold measurements in $\mathrm{n}-\mathrm{H}_{2}$ (a) and $\mathrm{p}-\mathrm{H}_{2}$ (b). The linear curve represents the pressure broadened line width $\Gamma(p)=97.3 \mathrm{p} \mathrm{MHz}$. The dotted curve is calculated by $(20)$, and the dashed curve by (21).

for $\mathrm{n}-\mathrm{H}_{2}$ and $\mathrm{p}-\mathrm{H}_{2}$, respectively, where for $\Gamma t^{\prime}>8$, the curves are dot-dashed to denote extrapolation. Equivalently, writing the threshold power as $P_{t h}\left(p_{0}, t_{0}^{\prime}\right)=E_{t h}\left(p_{0}, t_{0}^{\prime}\right) / t_{0}^{\prime}$, (13) can be used to calculate gain reduction data from the energy threshold data, which were measured as a function of pulse width. These gain reduction data points are plotted in Fig. 3 as a function of $\Gamma\left(p_{0}\right) \cdot t_{0}^{\prime}$ for both $\mathrm{n}-\mathrm{H}_{2}$ and $\mathrm{p}-\mathrm{H}_{2}$. From this figure, it thus appears that the same behavior of the gain reduction is measured for different experimental conditions, i.e., for different combinations of pressure and pulse width, if only the product $\Gamma t^{\prime}$ is equal for these conditions. Also, for the two different Raman transitions, $S_{0}(0)$ in $\mathrm{p}-\mathrm{H}_{2}$ and $S_{0}(1)$ in $\mathrm{n}-\mathrm{H}_{2}$, only small differences in gain reduction are observed. At pressures where the linewidth becomes Lorentzian, the dephasing time is given by $(\pi \Gamma)^{-1}$. Then, $\pi \Gamma t^{\prime}$ can be expressed in units of one dephasing time. The gain reduction curves (a) and (b) show that transient scattering may already appear for pulse widths as long as 100 dephasing times $\left(\Gamma t^{\prime} \simeq 32\right)$. For pulses shorter than 10 dephasing times, a Raman gain is measured which is less than half its value in steady state.

The experimental gain reduction is compared to the gain reduction calculated from the solutions for the transient Stokes amplitude, (7) and (8), of Carman et al. and Wang, respectively. In their approach, they assumed no pump depletion. In these threshold experiments, this condition is fulfilled. To

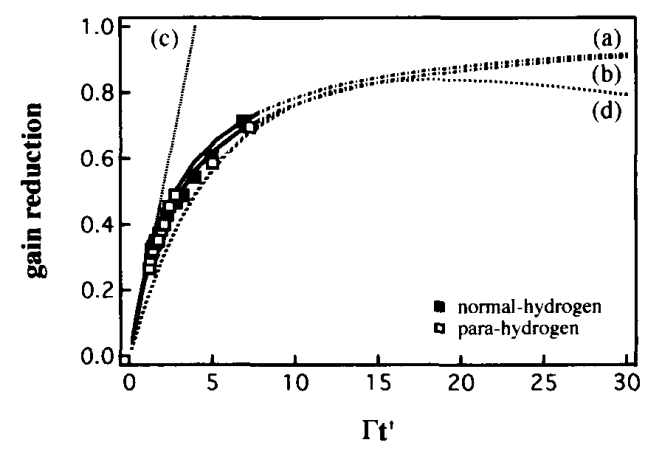

Fig. 3. Gain reduction as a function of the dimensionless parameter $\Gamma t^{\prime}$. Curves (a) and (b) show the experimentally derived gain reduction function (16) for $\mathrm{n}-\mathrm{H}_{2}$ and $\mathrm{p}-\mathrm{H}_{2}$, respectively, while (c) and (d) show the calculated gain reductions in the transient limit $\Gamma t^{\prime} \ll 1$ (Carman et al.), and in the transient regime $\Gamma t^{\prime}<g_{s}=$ (Wang), respectively. The measured gain reduction is shown as $(\square)$ for $n-H_{2}$ and $(\square)$ for $\mathrm{p}-\mathrm{H}_{2}$.

calculate the gain reduction from the analytical solutions, the transient Stokes gain is written as

$$
\ln \left[\frac{E_{s}}{E_{s 0}}\right]=g_{t} z=R \cdot g_{s} z
$$

where $E_{s}$ is the Stokes amplitude from either (7) or (8). Substituting the transient gain found by Carman et al., (7), into (22), the gain reduction factor is calculated as

$$
R \simeq \frac{2\left(g_{s} z \cdot \Gamma t^{\prime}\right)^{1 / 2}}{g_{s} z}
$$

Note that the calculated gain reduction still depends on the steady-state gain. To be able to compare the calculated gain reduction to the experimental result, the steady-state gain in the threshold experiments must be known. In these experiments, the power gain was constant but also transient. Therefore, the steady-state gain $g_{s} \cdot z$, which is related to the transient gain by the gain reduction, varies. Using the definition of the gain reduction, $R \cdot g_{s} z=g_{t} z$, (23) becomes

$$
R \simeq 4 \cdot \frac{\Gamma t^{\prime}}{g_{t} z}
$$

where $g_{t} z=16$ in these threshold experiments. Equation (24) is shown in Fig. 3 as curve (c). It shows excellent agreement with the experimental gain reduction for $\Gamma t^{\prime}<1$. The transient gain solution, (7), was assumed to be valid in the high gain limit $g_{s} z \cdot \Gamma t^{\prime} \gg 1$, and in the highly transient regime $\Gamma t^{\prime} \ll 1$. The experimental conditions can be compared to these requirements using (24) to rewrite the product $g_{s} z \cdot \Gamma t^{\prime}$ as $\left(g_{t} z / 2\right)^{2}$ which in these experiments equals 64 . Therefore, the experimental conditions fulfill the requirements of high gain. Equation (24) fits the experimental data for $\Gamma t^{\prime} \ll 1$, a condition which defines the highly transient regime. Apparently, this condition could even be relaxed to $\Gamma t^{\prime}<1$.

The transient Stokes amplitude calculated by Wang, (8), is now substituted into (22)

$$
R=\frac{1}{g_{s} z} \cdot \ln \left[\frac{E_{s}}{E_{s 0}}\right] \simeq \frac{1}{a^{2} / 4} \cdot \ln \left[\frac{e^{a \tau_{0}-\tau_{0}^{2}}}{\left(4 \pi a \tau_{0}\right)^{1 / 2}}\right] .
$$




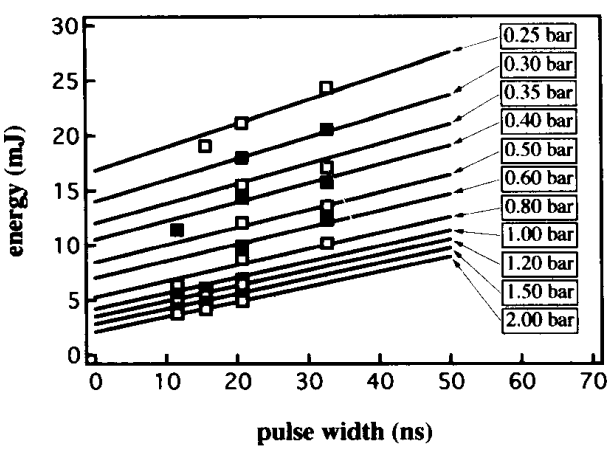

Fig. 4. Measured threshold energy as a function of pulse width for $n-H_{2}$ at gas pressures between 0.25 and 2.0 bar. The curves are calculated with (13) and (26).

As mentioned above, the steady-state gain was not a constant in the threshold experiments and must be replaced by $g_{t} z / R$. Equation (25) was solved for $R$ iteratively. Convergence was obtained in no more than 4 steps to less than $1 \%$ for a starting value of $R$ equal to unity. Equation (25) is represented by curve (d) in Fig. 3 . It is seen that the calculated gain reduction agrees well with the measured gain reduction for $\Gamma t^{\prime}<20$. Using the transient amplitude gain $\left(g_{t} z=16\right)$ and the measured gain reduction at $\Gamma t^{\prime}=20(R(20) \simeq 0.84)$, we find for the steady-state amplitude gain $g_{s} z \simeq 19$. Therefore, the regime $\Gamma t^{\prime}<g_{s} z$, for which Wang's solution was assumed to be valid, is also found experimentally.

\section{A. Threshold Energy as a Function of Pressure}

The linear dependence for the threshold energy upon pulse width, which is measured at pressure $p_{0}=1.2 \mathrm{bar}$, can be extended to other pressures. Using the pressure dependence of the gain reduction factor, the threshold energy $E_{t h}\left(p, t^{\prime}\right)$ was calculated for pressures in the experimental range. For two experimental conditions at threshold $\left(t^{\prime}, p\right)$ and $\left(t_{0}^{\prime}, p_{0}\right)$, for which the gain is reduced by the same factor, the threshold energy offset is written as

$$
E_{o}(p)=E_{o}\left(p_{0}\right) \cdot \frac{P_{t h, s}(p)}{P_{t h, s}\left(p_{0}\right)} \cdot \frac{\Gamma\left(p_{0}\right)}{\Gamma(p)}
$$

where (11) and (15) have been used. The threshold energy slope $P_{t h, s}(p)$ is found from (13). Fig. 4 shows the threshold energy curves, calculated with (13) and (26). Each curve represents the threshold energy versus pulse width for a fixed pressure. The obtained results from threshold measurements in $\mathrm{n}-\mathrm{H}_{2}$ as a function of pulse width for several pressures are in good agreement with the calculations, and therefore justify the concept of a gain reduction to describe transient scattering.

Typical calculated steady-state threshold powers for SRS in the room temperature 32 pass MPC at moderate high pressures ( 22 bar) for $\mathrm{p}-\mathrm{H}_{2}$ and $\mathrm{n}-\mathrm{H}_{2}$ are $0.11 \mathrm{MW}$ and $0.13 \mathrm{MW}$, respectively, and at 0.25 bar are $0.15 \mathrm{MW}$ and $0.22 \mathrm{MW}$, respectively. Typical calculated values for the energy offset are $1.9 \mathrm{~mJ}$ and $2.1 \mathrm{~mJ}$ at $2 \mathrm{bar}$, and $14 \mathrm{~mJ}$ and $17 \mathrm{~mJ}$ at 0.25 bar for $\mathrm{p}-\mathrm{H}_{2}$ and $\mathrm{n}-\mathrm{H}_{2}$, respectively. At pressures as low as $0.1 \mathrm{bar}$, an energy offset of $34 \mathrm{~mJ}$ is calculated for $\mathrm{p}-\mathrm{H}_{2}$.

\section{Summary AND CONCluSION}

SRS threshold experiments were performed in a multipass cell containing either $\mathrm{n}-\mathrm{H}_{2}$ or $\mathrm{p}-\mathrm{H}_{2}$ gas at room temperature and at gas pressures between 0.1 and 2 bar. Transient effects were observed as an offset in the steady-state threshold energy. For high gas pressures, the offset approaches zero describing the steady-state scattering. By the use of a gain reduction factor which can be derived from both threshold experiments and theory, excellent agreement was found between experiment and semiclassical theories in the transient and hypertransient regime. From the threshold energy measurements, the Raman linewidths of the $S_{0}(1)$ transition in $\mathrm{n}-\mathrm{H}_{2}$ and the $S_{0}(0)$ transition in $\mathrm{p}-\mathrm{H}_{2}$ were derived for gas pressures between 0.1 and 2 bar. The transitions show Dicke narrowing at low pressures, which exceeds the linewidth narrowing predicted by the simple sum of the effects of diffusional narrowing and pressure broadening.

\section{ACKNOWLEDGMENT}

The authors would like to thank H. Prins, G. Oudemijers, and A. Nieuwenhuis for their technical assistance.

\section{REFERENCES}

[1] E. E. Hagenlocker, R. W. Minck, and W. G. Rado, "Effects of photon lifetime on optical scattering in gases," Phys. Rev., vol. 154, pp. 226-233, 1967.

[2] Y. R. Shen, The Principles of Nonlinear Optics. New York: Wiley, 1984, ch. 10.

[3] R. L. Carman, F. Shimizu, C. S. Wang, and N. Bloembergen, "Theory of Stokes pulse shapes in transient stimulated Raman scattering," Phys. Rev., vol. A2, pp. 60-72, 1970.

[4] C. S. Wang, "Theory of stimulated Raman scattering," Phys. Rev., vol. 182, pp. 482-494, 1969.

[5] M. G. Raymer and I. A. Walmsley, "The quantum coherence properties of stimulated Raman scattering," in Progress in Optics, vol. XXIII, E. Wolf, Ed. Amsterdam: Elsevier Science, 1990, p. 259.

[6] M. G. Raymer and J. Mostowski, "Stimulated Raman scattering: Unified treatment of spontaneous initiation and spatial propagation," Phys. Rev., vol. A24, pp. 1980-1993, 1981 .

17) M. Abramowitz and I. A. Stegun, Handbook of Mathematical Functions. New York: Dover, 1970, ch. 9.

[8] W. R. Trutna and R. L. Byer, "Multiple pass Raman gain cell," Appl. Opt., vol. 19, pp. 301-312, 1980.

[9] G. D. Boyd, W. D. Johnston, and I. Kaminov, "Optimization of the stimulated Raman scattering threshold," IEEE J. Quantum Electron., vol. QE-5, pp. 203-206, 1969.

[10] R. L. Byer and R. L. Herbst, in Series in Optical Science, vol. 16, Y. R. Shen, Ed. Berlin: Springer, 1977, ch. 3.

[11] R. T. V. Kung, "Multiple pass stimulated Raman conversion with pump depletion," IEEE I. Quantum Electron., vol. QE-17, pp. 509-513, 1981.

[12] W. Koechner, Solid-State Laser Engineering, Series in Optical Sciences, vol. 1. Berlin: Springer, 1992, ch. 7

[13] R. Holmes and A. Flusberg, "Rotationally invariant theory of stimulated Raman scattering," Phys. Rev., vol. A37, pp. 1588-1596, 1987.

[14] M. D. Duncan, R. Mahon, J. Reintjes, and L. L. Tankersley, "Parametric Raman gain suppression in $\mathrm{D}_{2}$ and $\mathrm{H}_{2}$," Opt. Lett., vol. 11, pp. 803-805, 1986.

[15] A. Gavrielides and P. Peterson, "Diffractive Raman scattering in focused geometry," J. Opt. Soc. Amer, vol. B3, pp. 1394-1407, 1986.

[16] D. Herriott, H. Kogelnik, and R. Kampfner, "Off-axis paths in spherical mirror interferometers," Appl. Opt., vol. 3, p. 523, 1964.

[17] W. Demtröder, Laserspectroscopie, Grundlagen und Techniken. Berlin: Springer, 1993, ch. 3.

[18] J. I. Gersten and H. M. Foley, "Combined Doppler and collision broadening," J. Opt. Soc. Amer., vol. 58, pp. 933-937, 1968.

[19] K. D. van den Hout, P. W. Hermans, E. Mazur, and H. F. P. Knaap, "The broadening and shift of the rotational lines for hydrogen isotopes at low temperatures," Physica, vol. 104A, pp. 509-547, 1980. 
[20] A. Owyoung. "High-resolution cw stimulated Raman spectroscopy in molecular hydrogen," Opt. Lett., vol. 2, pp. 91-93, 1978.

[21] R. A. J. Keyser, J. R. Lombardi, K. D. van den Hout, B. C. Sanctuary, and H. F. P. Knaap, "The pressure broadening of the rotational Raman lines of hydrogen isotopes," Physica, vol. 76, p. 585, 1974.

R. J. Heeman, photograph and biography not available at the time of publication.

H. P. Godfried, photograph and biography not available at the time of publication. 